\title{
Unusual case report of an intraspinal foreign body presenting as a meningocoele
}

\author{
Sunilkumar C Baranwal', Suniti Kumar Saha², Sibaji Dasgupta', B V Sandeep ${ }^{1}$ \\ ${ }^{1}$ Senior Registrar, Department of Neurosurgery, Nilratan Sircar Medical College and Hospital, Kolkata, India, \\ ${ }^{2}$ Professor and Head, Department of Neurosurgery, Nilratan Sircar Medical College and Hospital, Kolkata, India
}

Background: Non missile penetrating foreign body of the lumbo-sacral region presenting as a pseudomeningocele is an extremely rare condition. No significant data regarding this is available in our hand till date. We are reporting here a unique case of penetrating foreign body spine with pseudomeningocele presenting as lumbo-sacral swelling in a $1 \frac{1 / 2}{2}$ year old child. Case Description: A $1 \frac{1}{2}$ year male child presented with painless, low back midline swelling gradually increasing in size for 6 months. Swelling increased in size on crying and coughing. There was no external scar mark. Plain radiography revealed presence of a long slender radio-opaque foreign body in the spinal canal. Non- contrast computed tomography showed that the foreign body had penetrated the spinal canal at the level of L5 - S1 with a cystic space of CSF attenuation overlying it and extending into subcutaneous plane. Patient was explored through a transverse incision overlying the swelling. After opening the sac, the foreign body was found impacted between L5 and S1 lamina with CSF around the foreign body. The foreign body, a sewing needle was retrieved without disturbing its original trajectory. The narrow neck of sack was communicating with the spinal canal through a small dural defect which was closed and the sac was excised. Conclusion: A careful and meticulous approach is required from the surgeons point of view while taking a history, clinically assessing and performing surgery for patients presenting with acquired spinal swelling with such a unique etiology.

Access this article online

Website:

http://nepjol.info/index.php/AJMS

DOI: 10.3126/ajms.v7i4.14084

E-ISSN: 2091-0576

P-ISSN: 2467-9100

Key words: Lumbosacral swelling, Foreign body, Pseudomeningocoele

\section{INTRODUCTION}

Non-missile penetrating spinal injuries are very rare and may present as a spectrum of neuro deficit, abscess or cerebrospinal fluid (CSF) leak etc. Extradural collections of CSF (Pseudomeningocele) are commonly iatrogenic, resulting from incidental durotomies during spinal surgery and in our knowledge no case has been reported with penetrating spinal injury. We are reporting here a unique case of penetrating intraspinal foreign body with pseudomeningocele presenting as a lumbar swelling in one and a half year old child.

\section{CASE REPORT}

A one and half-year-old male child presented with painless, low back midline swelling gradually increasing in size for 6 months. Clinically it was a soft, cystic, fluctuant and transilluminant lumbo-sacral swelling about $5 \mathrm{~cm}$ in diameter. The swelling increased in size on crying and coughing. There was no external scar mark. Findings on neurological examination were within normal limits. According to the parent who is tailor by profession, there was no history of any trauma. They had noticed a red, indurated swelling over the lower back 6 months back, which subsided on some medication by local doctor. But it grew in size to attain the present form. Plain radiography revealed presence of a long slender radio-opaque foreign body in the spinal canal. Non- contrast computed tomography (NCCT) (axial cuts) showed that the foreign body had penetrated the spinal canal at the level of L5-S1 with a cystic space of CSF attenuation overlying the same and extending into subcutaneous plane (Figure 1). Patient 
was operated upon with a longitudinal incision of $7 \mathrm{~cm}$ overlying the swelling. After wide opening of the sac, the foreign body was detected impacted between L5 and S1 lamina with the foreign body encased in a CSF pocket. The foreign body was retrieved gently without disturbing its original trajectory. It was a sewing needle. The narrow neck of sack was communicating with the spinal canal through a small dural defect which was closed (Figure 2). Sac was excised and histopathological examination (HPE) of the sac wall showed it to be of non-specific inflammatory origin.

His postoperative course was uncomplicated and was discharged seven days later after removal of the sutures.

\section{DISCUSSION}

Penetrating injuries to the spine are rare causes of spinal injury after Road traffic accident and fall from height.

It are of two categories

1) Missile penetrating spinal injuries (MPSI) and

2) Non-missile penetrating spinal injuries (NMPSI) varieties.

Further these two categories can be subdivided into two types: Metallic and Non metallic.

Gunshot injury is the most common type of PSI.

Other types of non-missile foreign bodies (FB), though rare, are also responsible for the penetrating injuries to the spine, for example knife, fragment of glass, metallic splinters, nail, and sugar cane.

In our case the FB retrieved was a sewing needle.

On physical examination, most superficial FB can be visualized or palpated easily and usually have an injury track leading to the splinter that facilitates its detection and removal. Deeper splinters may be difficult to detect and sometimes without history of FB exposure. The only clue to the presence of retained FB may be presence of a swelling, tenderness, mass, draining sinus or a soft tissue infection such as cellulitis, abscess, etc. The composition of the FB dictates the reaction of the tissues to the splinter. ${ }^{1}$

In our case there was no history of any trauma and no obvious entry point visualized on clinical examination. The parent noticed a red, indurated swelling which decreased in size on medication, pointing to the possibility of initial abscess/inflammation.

Standard radiographs are the most practical means of screening for a radio-opaque FBs. Almost all glasses are

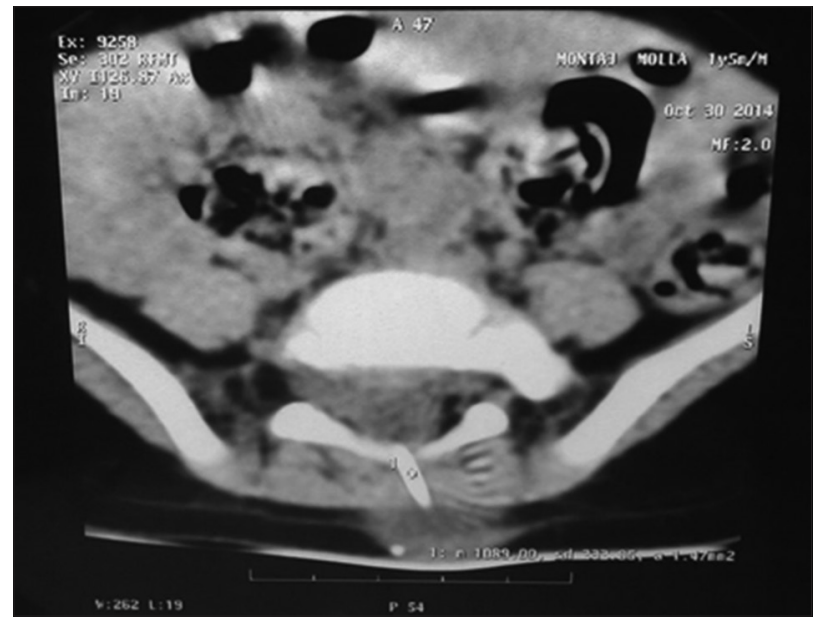

Figure 1: NCCT (axial and sagittal cut) showing foreign body that had penetrated the spinal canal with a cystic space of CSF attenuation overlying the same and extending into subcutaneous plane

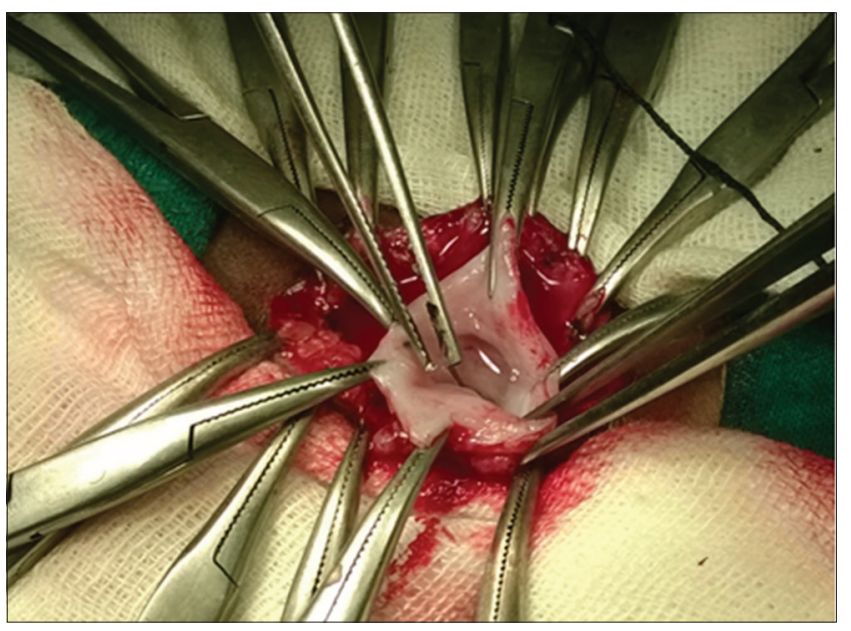

Flgure 2: Per op picture showing foreign body which is a sewing needle inside the sac and the narrow neck of sack communicating with the spinal canal through a small dural defect

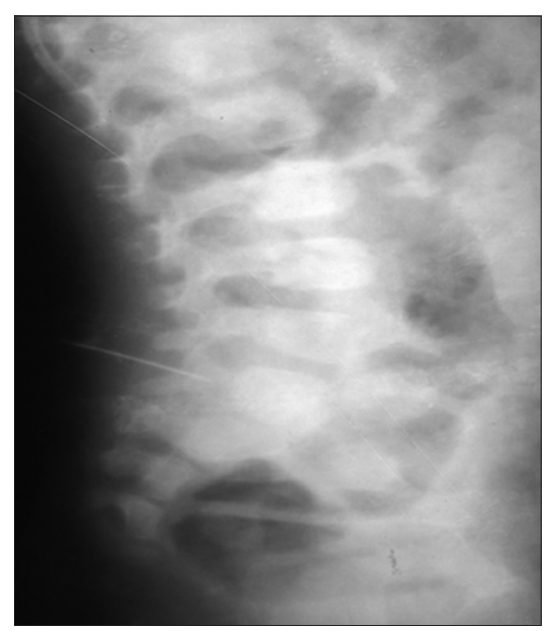

Figure 3: $\mathrm{X}$ ray of the lumbosacral spine showing a long slender foreign body at level of L5-S1 
radiodense, and glass FBs as small as 0.5 to $2 \mathrm{~mm}$ can be detected easily on plain radiographs. NCCT scanning is a good investigating modality for the patients with NMPSI considering its ability to detect retained foreign body, spinal or paraspinal hematoma and bony fragments. ${ }^{2}$

Magnetic resonance imaging (MRI) detects many FBs that may be missed on radiographs but its use is controversial as it may be associated with movement and heating of retained metallic objects.

On plain radiography presence of a long slender radioopaque FB in the spinal canal was revealed. Suspecting a metallic foreign body we preferred a NCCT, which further delineated the foreign body and its extension into the spinal canal.

Neurological deficit may occur immediately or in delayed fashion. Immediate injury is caused by physical damage to the neural tissue, in-driven bone fragments, vascular injury or countercoup mechanism. Delayed neurological deficit may result from a retained weapon, infection, edema, or CSF leak. ${ }^{3}$ Peacock et al. documented $4 \%$ spontaneous subsidence of CSF leak. ${ }^{4}$ There was no neurological deficit on clinical examination, but cystic lumbo-sacral swelling which was initially mistaken as meningocele. Pseudomeningocele is an extradural collection of CSF that results from a dural breach. This extradural fluid may be contained in an arachnoid-lined membrane or a fibrous capsule. ${ }^{5}$ The histopathology of sac excised showed nonspecific inflammation, no arachnoid lining was seen.

By far, the majority of pseudomeningoceles are iatrogenic, resulting from incidental durotomies during spinal or intradural surgery with incidental between 0.3 and $13 \%{ }^{6}$ But traumatic and congenital causes may also be implicated. ${ }^{7}$ This is rare case of pseudomeningocele following trauma with a retained foreign body.

Pseudomeningoceles are caused by the egress of CSF into the soft tissue, but not through the skin closure, eventually forming a fibrous capsule. When the dura is breached but the arachnoid remains intact, the arachnoid can become herniated through the dura and an arachnoid-lined sac becomes the pseudomeningocele. The CSF pulsations can push the pseudomeningocele into the soft tissue and affect its size, shape, and location. ${ }^{8}$

As stated previously, pseudomeningoceles are often asymptomatic; however, they can be diagnosed based on a subcutaneous or subfascial fluid collection that increases with Valsalva maneuvers such as sneezing and coughing. Seroma, liquefied hematoma, wound infection, and abscess should be ruled out to reach a definite diagnosis.'
Similar clinical picture lead to the initial diagnosis of meningocele, which on histopathology was confirmed as pseudomeningocele.

Most of the studies have suggested that surgical exploration should be considered in patients with progressive neurological deficits, when there is radiographic evidence of neural compression due to retained foreign material, bone fragment or soft tissue or persistent CSF leakage and pain. ${ }^{10}$

\section{CONCLUSION}

Pseudomeningocele is a rare condition and majority is iatrogenic, resulting from incidental durotomies during spinal or intradural surgery. Spinal foreign body presenting as a pseudomeningocele is an extremely rare condition. Though the history of significant trauma may be missed by the patient or the patient's relatives, a careful and meticulous history taking concerning apparently insignificant aspects like occupation is required. While managing an acquired lumbosacral swelling in a child, a possibility of intraspinal foreign body should be kept in mind and one must examine thoroughly including neurological assessment and palpate the swelling carefully as there lies every chance of imminent neurological deterioration during examination. Meticulous dissection and properly layered closure can lead to early and uneventful post operative period and successful recovery.

\section{REFERENCES}

1. Lammers RL and Magill T. Detection and management of foreign bodies in soft tissue. Emerg Med Clin North Am 1992; 10: 767-781.

2. Mizel MS, Steinmetz ND and Trepman E. Detection of wooden foreign bodies in muscle tissue: Experimental comparison of computed tomography, magnetic resonance imaging, and ultrasonography. Foot Ankle Int 1994; 15: 437-443.

3. Kulkarni AV, Bhandari M, Stiver S and Reddy K. Delayed presentation of spinal stab wound: Case report and review of literature. J Emerg Med 2000; 18: 209-213.

4. Peacock WJ, Shrosbree RD and Key AG. A review of 450 stab wounds of spinal cord. S Afr Med J 1977; 51(26): 961-964.

5. Miller PR and Elder FW. Meningeal pseudocysts (meningocele spurius) following laminectomy. Report of ten cases. J Bone Joint Surg Am 1968; 50: 268-276.

6. Lee KS and Hardy IM. Postlaminectomy lumbar pseudomeningocele: Report of four cases. Neurosurgery 1992; 30: 111-114.

7. Barberá J, Broseta J, Argüelles $F$ and Barcia-Salorio JL. Traumatic lumbosacral meningocele. Case report. J Neurosurg 1977; 46: 536-541.

8. Tsuji H, Handa N, Handa O, Tajima $G$ and Mori K. Postlaminectomy ossified extradural pseudocyst. Case report. J Neurosurg 1990; 73: 785-787. 
9. O'Connor D, Maskery N and Griffiths WE. Pseudomeningocele nerve root entrapment after lumbar discectomy. Spine 1998; 23: 1501-1502.
10. Shahlaie K, Chang OJ and Anderson JT. Nonmissile penetrating spinal injury. Case report and review of the literature. J Neurosurg Spine 2006; 4: 400-408.

\section{Authors Contribution:}

SCB - Concept and design of the study, reviewed the literature, manuscript preparation and critical revision of the manuscript; SKS - Patient admitted and operated under his care; SD - Reviewed the literature and helped in preparing first draft of manuscript; SBV - Reviewed literature, helped in preparing first draft of manuscript and helped in editing the manuscript. 